\title{
THE DANGERS OF STREPTOMYCIN WITH PARTICULAR REFERENCE TO THE VESTIBULAR TOXIC EFFECTS
}

By D. RANGeR, F.R.C.S. Assistant E.N.T. Surgeon, Middlesex Hospital and London Chest Hospital

There is some risk attached to the administration of all antibiotics and none of them should be given without an adequate reason: Some antibiotics may cause death on rare occasions but apart from this extremely rare and exceptional complication, most of the side-effects are reversible and are followed sooner or later by complete recovery. Streptomycin, however, provides an exception to this general pattern, because its commonest toxic effects are frequently permanent and disabling. This places streptomycin in a special category among the antibiotics and calls for a very critical appraisal of its need before starting its administration to a patient.

Because the toxic reactions of streptomycin often affect only the special sense organs of balance and hearing, patients developing complications from this therapy may be referred to special departments for diagnosis and treatment, and it is possible that in some cases the clinician who prescribed the drug does not become fully aware of the serious and permanent nature of the disability which has been produced. This is especially likely to happen when the antibiotic has been given for an acute or minor infection, where follow-up of the original condition is not needed for long, and yet these are the patients in whom it is most important that the full implications of treatment should be appreciated by the prescriber. In severe tuberculosis and some other infections, loss of balance and even deafness may have to be accepted as a necessary price to pay for recovery, and in some of these cases treatment may have to be continued in spite of the development of these complications. On the other hand it is an entirely different matter if a patient loses permanently his sense of balance as a result of treatment for a minor condition which would certainly have resolved without recourse to streptomycin.

In terms of percentages there may seem to be little risk of permanent disability resulting from the administration of moderate doses of streptomycin, but there is certainly some danger of this happening and in assessing the risks of any treatment the severity of the complications which may occur is even more important than their statistical incidence. A slight risk of a severe permanent disability must be a far greater obstacle to the use of a drug than a considerable chance of the occurrence of a slight temporary setback.

\section{Toxic Effects}

Fatal cases of encephalopathy and encephalo meningitis have been reported as a result of treato ment with streptomycin but these cases have been rare and at least some of them have resulted from excessive dosage in the presence of impaired renal function. Exfoliative dermatitis and other skin lesions have also occurred and people who handle the drug frequently are liable to develop contact dermatitis. Early batches of streptomycin often produced histamine-like reactions from the presence of impurities but these are seldom seen now.

Unfortunately, in contrast to the above complications, the incidence of damage to the labyrinthine system shows no sign of diminishing and some clinicians who have been prescribing streptomycin regularly for several years think that these complications from small doses of the drug are actually becoming more common. They will now be considered in greater detail.

\section{Ototoxicity}

All forms of streptomycin known at present have a curious liability to damage the eighth nerve system but there is some variation in the pattern of 0

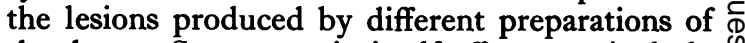
the drug. Streptomycin itself affects particularly $\stackrel{\mathcal{D}}{\rightarrow}$ the balancing mechanism and moderate doses $T$ seldom produce tinnitus or deafness. Dihydrostreptomycin, on the other hand, has a selective damaging effect on the cochlear system, thereby $\mathbb{\Omega}$ 
producing deafness, and is unlikely to cause vertigo except with large doses. In a proportion of patients this deafness from dihydrostreptomycin develops some time after the drug has been stopped.

Deafness is an even greater handicap than giddiness and for that reason streptomycin is to be preferred to dihydrostreptomycin. Claims have been made that mixtures of the two preparations are less toxic than an equivalent dose of either form alone, but both deafness and giddiness have occurred as a result of using the combination. Streptomycin seems to be the best form of the drug available to date and this paper is concerned mainly with this preparation.

Experimental studies in animals have shown that stieptomycin may damage the vestibular end organs or the vestibular nuclei in the brain stem and both regions may be involved. There is still dispute as to the site of the lesion which is responsible for the toxic effects in man and it is possible that there is a variation in different cases and that this explains some of the differences observed in the clinical pattern.

With a toxic reaction of this nature it might be expected on theoretical grounds that the effects on the vestibular system would always be bilateral and symmetrical. In practice this is not so and it is not uncommon to find that the function of one labyrinth has been destroyed while the other may still be functioning normally. Of course lesser degrees of asymmetry are also seen as well as symmetrical lesions.

\section{Dose Necessary to Produce Toxic Effects}

It has been known almost since its introduction into clinical use that streptomycin has a selective damaging effect on the eighth nerve system and the reason for this is still not known. In the early stages streptomycin was used mainly in the treatment of serious tuberculous infections, such as tuberculous meningitis and miliary tuberculosis, and was given in large doses. Later it was used in other less serious forms of tuberculosis and although the daily and weekly dose was reduced the patient still received a large total dose of the drug during the whole course of treatment. Even in these cases toxic effects were by no means invariable, and it is well known that many patients will tolerate a dose of $\mathrm{I}$. per day for several months without any apparent untoward effects. For these reasons it was perhaps natural that many clinicians should have come to regard ototoxicity as something which occurred only with large doses of the drug.

When streptomycin started to be used on a large scale, often for the treatment of minor infections, it became more evident that in some patients dis- turbance of balance could occur with very small doses of the drug and cases have now been reported of ototoxic effects occurring when the total dose has not exceeded $3 \mathrm{~g}$., $4 \mathrm{~g}$. or $5 \mathrm{~g}$. spread over the same number of days. That these cases are not uncommon was shown in 1957 when Cawthorne and Ranger reported 22 cases seen personally in which eighth nerve damage had occurred with a total dose of $16 \mathrm{~g}$. or less of streptomycin. In 13 of these cases the daily dose had not exceeded $\mathbf{I} g$. and in 12 cases the total dose given was $10 \mathrm{~g}$. or less.

Although this untoward reaction to small doses of streptomycin indicates that the patients concerned are 'hypersensitive' to the toxic effects of the drug there is nothing to indicate that in them the response is of a different nature from that occurring in patients who have received hundreds of grammes before developing symptoms. The effect on the labyrinth in both groups is an impairment, or complete loss, of vestibular function and also there is no dividing line between ' small' and 'large' doses. Cases of ototoxicity are seen as a result of the administration of a total of $5 \mathrm{~g}$., 10 g., 20 g., 50 g. and 100 g.-in fact with almost any dose at all-and the absence of toxic effects after a few grams have been given is no indication that the drug can be continued safely.

It must be accepted that there is some risk attached to the administration of any streptomycin and that at present it is quite impossible to predict in which patients it will occur or with what dose. However, it is known that the higher the daily amount, the more likely are toxic effects to occur. It is also known that the administration of streptomycin is especially hazardous in the presence of impaired renal function. In some cases of renal disease it has been shown that a dose of $0.2 \mathrm{~g}$. streptomycin will produce blood levels as high as those found after the administration of $\mathrm{r} .0 \mathrm{~g}$. to people with normal urinary excretion and obviously it is unnecessary as well as unwise to give full doses to such patients. In any case of known renal disease streptomycin should be avoided if at all possible, but if it must be used fractional doses may suffice and an estimation of blood levels is desirable.

\section{Symptoms and Signs of Ototoxicity}

The degree of vestibular damage and the' speed with which it develops profoundly influence the effects produced on the patient and the symptoms of ototoxicity vary considerably because of this. Age is also an important factor because of its influence on the ability of the patient to compensate for the impairment of vestibular function by special development of the visual and kinaesthetic senses. A young person usually develops a considerable degree of compensation whereas an 
elderly patient often fails to compensate to any extent, and may remain crippled afterwards, needing a walking stick even on level ground and finding it impossible to cope with uneven surfaces or with the difficult task of balancing in a moving vehicle such as a train.

A sudden complete failure of vestibular function produces severe vertigo and nystagmus and in the early stages there may also be vomiting. This picture is sometimes seen with streptomycin toxicity but usually the damage is more gradual and the effects less severe. In that event a patient who is walking about may first notice only slight unsteadiness on sudden movements or on stooping. Patients in bed often notice difficulty in reading and in turning over to get things from the bedside locker. Many patients with early toxicity experience sensations which they find difficult to explain in words and such expressions as 'a feeling of unreality,' ' light headedness,' ' a muzzy feeling in the head 'and others, as well as the more definite expressions of 'giddiness' and 'dizziness' may all indicate vestibular damage.

Because of the circumstances mentioned above which provoke symptoms in some patients, movements such as turning over in bed or stooping in the case of up-patients can be used as test procedures for the detection of vestibular damage especially if they are performed with the eyes shut. Observation of the ability to walk with the eyes shut is a very valuable test and perhaps movements such as these should be tested daily from the commencement of treatment even in the absence of symptoms.

A more delicate and quantitative assessment of vestibular function is provided by the differential caloric test described by Cawthorne, Fitzerald and Hallpike (1942) and this will need to be used in doubtful cases and in assessing deterioration or recovery of labyrinthine function. It is also most important in excluding functional disturbances and no patient on streptomycin should ever be diagnosed as suffering from hysterical giddiness without the evidence of a caloric test.

\section{Prophylaxis and Treatment of Ototoxicity}

It has already been stressed that streptomycin should not be prescribed without considering the possible risks of toxic effects in relation to the possible advantages of its use and it has also been pointed out that the smaller the daily dose compatible with therapeutic effectiveness, the smaller is the chance of toxic reactions, especially in the presence of renal disease.

If a patient receiving streptomycin develops any symptoms or signs which suggest the possibility of toxicity, the position must be reviewed im- mediately and the necessity for using streptomycin reconsidered. At this stage the damage may only be partial and the symptoms slight and if the streptomycin can be stopped then the disability may not be too great. Also, although in many cases the effects on the vestibular system are $\overline{0}$ permanent, nevertheless full recovery of vestibular $\bar{D}$ function does occur in some people and this is more likely to happen if the drug is stopped immediately at the first symptom when the lesion may be only partial rather than later when the damage has become greater.

In spite of what has been said above it sometimes happens that when caloric reactions are first tested, no vestibular response at all is obtained and it $\vec{C}$ might be considered that under these circum- 3 . stances the streptomycin could be continued of indefinitely without any further harm resulting. Unfortunately this is not so and the following points seem to be relevant.

I. Complete loss of the caloric response does not necessarily indicate absolute loss of vestibular function.

2. Even if the caloric responses are completely lost it is still possible that some recovery could occur but further streptomycin might well ing $\vec{v}$ terfere with the chances of recovery.

3. Not enough is known about the exact site of action of streptomycin in producing its toxie effects in man, and it is possible that continuing the streptomycin might increase the disability by

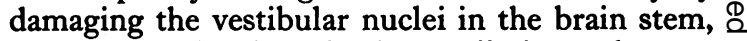
whereas previously only the vestibular end organ $\overrightarrow{\vec{B}}$ itself may have been affected. For these reasons it $\frac{0}{3}$ is considered that whatever is the degree of damage when ototoxicity is first diagnosed the streptomycin should be stopped unless the patient's infection demands that streptomycin must be continued.

The treatment of established ototoxicity depends on the extent of the damage to the vestibular system and on the degree of compensation which has already occurred. If the damage is slight and $\frac{9}{2}$ and symptoms not great, no treatment is required $D$ apart from reassurance and encouragement. With severe disturbances of balance the nature of $N$ the disability should be explained to the patient and as soon as possible he should be started on $\mathcal{N}$ balancing exercises, such as those described by $\omega$ Cawthorne and Cooksey (1946).

\section{BIBLIOGRAPHY}

CAWTHORNE, T. (1946), Proc. roy. Soc. Med., 39, 270. CAWTHORNE, T., FITZGERALD, G., and HALLPIKE, $C . s . \bar{o}$ (1942), Brain, 65, 115. CAWTHORNE, T., and RANGER, D. (1957), Brit. med. Y., 1, 1444. COOKSEY, F. S. (1946), Proc. roy. Soc. Med., 39, 273. 